\title{
Characteristics of Cardiac Rhythm and Conduction Abnormalities Diagnosed in Mobile Emergency Units in Central Romania
}

\author{
Aurelian Lucian Masalar ${ }^{1}$, Eugen Linga ${ }^{2}$, Sorin Crișan³, Dan Rădulescu³, Elena Buzdugan³ \\ ${ }^{1}$ Clinic of Pediatrics 2, Department of Pediatric Cardiology, Cluj-Napoca, Romania \\ 2 "Octavian Fodor" Regional Institute of Gastroenterology and Hepatology, Department of Intensive Care - Cluj-Napoca, Romania \\ ${ }^{3}$ Medical Clinic V, "Iuliu Hațieganu" University of Medicine and Pharmacy, Cluj-Napoca, Romania
}

\section{ABSTRACT}

Background: Cardiac arrhythmias and conduction abnormalities are frequently identified by emergency medical service staff in patients requesting emergency services. Methods: We conducted a prospective observational study that aimed to analyze the characteristics of rhythm and conduction abnormalities in prehospital settings, in patients who requested emergency medical assistance for symptoms indicative for an arrhythmia or conduction abnormalities. Results: From 180 patients included in the study ( 90 from a tertiary and 90 from a non-tertiary medical center), $92.78 \%$ presented an arrhythmia and $15.56 \%$ a conduction abnormality. A clear etiology was found in only $29.44 \%$ of cases. Ischemic patients presented a higher rate of atrial fibrillation $(\mathrm{AF})(\mathrm{p}<0.0001)$, while sinus tachycardia was more common in non-ischemic subjects $(p=0.02)$. Patients younger than 50 years of age presented higher rates of sinus tachycardia ( $\mathrm{p}<0.0001$ ) and right bundle branch block ( $\mathrm{p}=0.001)$, while those older than 50 years presented higher rates of AF $(\mathrm{p}<0.0001)$ and ventricular extrasystole $(\mathrm{p}=$ 0.014). There were no differences regarding the type of arrhythmia diagnosed in emergency settings, neither between genders or provenance environments, nor between regions served by a tertiary versus non-tertiary base station hospital. Conclusions: The most frequently diagnosed arrhythmias in prehospital settings were atrial fibrillation and sinus tachycardia, while atrioventricular blocks were the most frequent conduction disturbances. Young patients more frequently present sinus tachycardia or right bundle branch blocks, while older subjects are more prone to develop ischemia-related AF and ventricular extrasystole. The lack of any significant differences between systems served by a tertiary vs. non-tertiary medical center indicates an efficient emergency system of care regardless of the geographical region.

Keywords: rhythm and conduction abnormalities, mobile emergency units, prehospital ECG, tertiary and non-tertiary hospital

\section{ARTICLE HISTORY}

Received: September 10, 2017

Accepted: November 15, 2017

\section{CORRESPONDENCE}

Elena Buzdugan

Str. Victor Babeș Nr. 8 400000 Cluj-Napoca, Romania

Tel: +40 745387671

E-mail: buzelena@yahoo.com 


\section{INTRODUCTION}

Cardiac arrhythmias and conduction abnormalities are frequently diagnosed by emergency medical service (EMS) staff in patients calling an ambulance., ${ }^{1,2}$ The clinical manifestations of cardiac rhythm disturbances are usually benign and vary from asymptomatic forms or sensation of dizziness, to palpitations or syncope. These symptoms are mainly caused by paroxysmal supraventricular tachycardia, atrial fibrillation (AF), sinus bradycardia, sinus tachycardia, or atrioventricular blocks (AVB).

In emergency settings, differentiation between ventricular and supraventricular arrhythmia is mandatory for a proper management. ${ }^{3}$ In most of the cases, prehospital diagnosis of tachy- and bradyarrhythmias is easily achieved based on monitor tracings. However, on-site recording of a 12-lead ECG is still required in certain cases, especially when an acute coronary syndrome (ACS) is suspected. ${ }^{4}$

Prehospital recording of the ECG has been indicated before initiation of the targeted treatment outside the hospital, and for activation of catheterization laboratory in case of ACS. The use of systems for ECG transmission to centers with cath labs has led to a significant decrease in false positive field activations for ST-segment elevation myocardial infarction (STEMI). ${ }^{5}$ On the other hand, the lack of a 12-lead ECG in prehospital settings will lead to an increase in reperfusion times and also to a higher rate of denied reperfusion treatment in STEMI patients. ${ }^{6}$ Prehospital ECG recordings and the use of telemetry-based ECG interpretation are important not only for STEMI patients, but also for patients referred for syncope, stroke, or any suspicion of arrhythmia. ${ }^{7-9}$

The most severe clinical expression of cardiac arrhythmias is represented by sudden cardiac death (SCD). The estimated incidence of SCD is around 50-100 cases per 100,000 persons per year, and approximately $80 \%$ of the cases are caused by ventricular arrhythmias such as ventricular tachycardia and fibrillation..$^{10}$ SCD is more frequent in male subjects, and its frequency increases with age, mainly due to a higher prevalence of coronary artery disease and to the physiological aging process of the myocardium. ${ }^{11}$ The etiology of SCD is variable among age groups, as in younger patients there is a predominance of cardiomyopathies and channelopathies, myocarditis and recreational drug misuse, while in older subjects there is a shift towards degenerative disease, coronary artery disease, valvular disorders, and congestive heart failure. $^{12-14}$

Determination of the etiology of rhythm and conduction abnormalities is of utmost importance, as it allows proper treatment. For instance, in patients with acute myocardial ischemia and related arrhythmic complications, urgent revascularization therapy has led to a significant decrease in arrhythmia-related complications and an increase in survival rates. ${ }^{15}$ Rhythm and conduction abnormalities are also triggered by various nonischemic causes, varying according to age, gender, and demographics. In younger patients, besides non-ischemic cardiac conditions (cardiomyopathies, myocarditis, channelopathies), non-cardiovascular etiological factors include recreational drug misuse; these lead to increased sympathetic activity and increased myocardial excitability. ${ }^{12,16}$ Other causes for secondary arrhythmias include endocrinological conditions, psychiatric disorders and their treatments, intoxications, electrolyte imbalances, trauma that leads to increased sympathetic activity, or infectious disease..$^{12,17-20}$

\section{STUDY AIM}

The primary objective of the study was to analyze the characteristics of rhythm and conduction abnormalities in prehospital settings, in patients who requested emergency medical assistance for symptoms indicative for an arrhythmia or conduction abnormalities and were transported to the Emergency Unit of the base station hospital.

The secondary objectives of the study were: (1) to analyze the age, gender, and provenience-related differences in the rhythm and conduction abnormalities; (2) to study the frequency of different types of arrhythmia in the subgroup of ischemic etiology versus the subgroup with non-ischemic etiology; (3) to study if there were any differences in arrhythmias and conduction abnormalities diagnosed in the ambulance, between two sample regions in Central Romania: one served by a tertiary and one by a non-tertiary base station hospital.

\section{MATERIALS AND METHODS}

The study was a prospective observational one, conducted between 2014 and 2016, and included 180 patients from two neighboring counties, one being served by a tertiary base station hospital and the other by a non-tertiary one. All the included patients had solicited emergency medical assistance for symptoms indicating an arrhythmia or conduction abnormality, had been evaluated in the mobile emergency medical unit and transported to the Emergency Unit (EU) of the base station hospital.

The study was conducted in accordance with the principles stipulated in the Declaration of Helsinki. All the 
subjects agreed with processing of their data, and if they were unable to give informed consent, approval was obtained from their legally authorized representative. The study procedures were carried out following approval of the institution where the patients were treated.

The study population included all patients who required emergency medical assistance for the following complaints: palpitations, dizziness, syncope, loss of consciousness, or cardiac arrest. A 12-lead ECG of continuous ECG monitoring was achieved for all patients included in the study. Arrhythmias or conduction abnormalities of any cause (cardiac or non-cardiac) were recorded during monitorization in the ambulance.

Patients in whom ECG tracing could not be clearly interpreted due to motion artifacts, excessive weight or pilosity, or due to errors caused by the ECG recording equipment were excluded from the study. Also, patients who had been unconscious or in an altered mental state, with absent witnesses, with no possibility for anamnesis and therefore no possibility for analysis of the cause, associated symptoms or medical history, were also excluded from the study.

All included subjects underwent continuous monitorization in the ambulance, with the use of the Corplus monitoring equipment (GS Elektromedizinische Gerate G. Stample GmbH, Germany), which can provide live ECG tracings, 12-lead ECG for diagnosis, C-reactive protein analysis, pulse oximetry, extended oximetry $\left(\mathrm{SpO}_{2}, \mathrm{SpHb}\right.$, SpMet), capnometry $\left(\mathrm{CO}_{2}\right)$, temperature recording, as well as invasive and noninvasive arterial blood pressure monitoring.

The research was structured in several sub-studies, according to the secondary objectives.

The first sub-study aimed to characterize the different patterns of cardiac arrhythmia in the subgroup of patients $\leq 50$ years old $(n=59)$ and the subgroup of patients $>50$ years old $(n=121)$.

The second sub-study included 53 patients in whom the etiology of cardiac arrhythmia was identified and aimed to evaluate the differences in arrhythmia type between the ischemic $(n=15)$ and non-ischemic $(n=38)$ subgroups.

The third sub-study was a comparative analysis on the characteristics of rhythm and conduction disturbances diagnosed in mobile emergency units activating in two different regions, one served by a tertiary and the other one by a non-tertiary base station hospital. This sub-study aimed to see if there are any system-related differences in the emergency care in relation to diagnosis of these types of cardiac arrhythmia.

Data analysis was performed using the R Project for statistical computing software, version 3.2.3 (Bell Labo- ratories, New Jersey, USA, 2015-12-10). The continuous variables were expressed as mean \pm standard deviation, or median when appropriate, and categorical variables were expressed as integer and percentage values. The statistical significance of the data analysis was set at an alpha coefficient of 0.05 , with a confidence interval of $95 \%$.

\section{RESULTS}

\section{STUDY POPULATION}

In total, 180 patients were included in the study, 90 from a region served by a tertiary base station hospital and 90 from a non-tertiary center. From the total number of cases, an identifiable cause was found in the ambulance in only $29.44 \%(n=53)$ of patients. The demographic characteristics of the study population, as well as the type of rhythm and conduction abnormalities are listed in Table 1. From the 180 patients included in the study, 92.78\% $(n=167)$ presented a type of arrhythmia or asystole, while $15.56 \%(n=28)$ presented atrioventricular or interventricular conduction abnormalities, most frequently AVBs (Table 1).

\section{RHYTHM AND CONDUCTION ABNORMALITIES IN ASSOCIATION WITH AGE, PROVENANCE, AND GENDER}

Subjects aged over 50 years presented more frequently $\mathrm{AF}(\mathrm{p}<0.0001)$ and ventricular extrasystole $(\mathrm{p}=0.014)$, while the group younger than 50 years presented in a sig-

TABLE 1. Patient demographics and rhythm and conduction abnormalities encountered in the study population

\begin{tabular}{lc}
\hline Patient demographics & \\
Mean age (mean \pm SD; range) & $57.87 \pm 19.96(17-88)$ years \\
Gender (male) & $45 \%(\mathrm{n}=81)$ \\
Provenance (rural) & $51.11 \%(\mathrm{n}=92)$ \\
Type of arrhythmia & \\
Sinus bradycardia & $12.22 \%(\mathrm{n}=22)$ \\
Sinus tachycardia & $21.11 \%(\mathrm{n}=38)$ \\
Supraventricular extrasystole & $9.44 \%(\mathrm{n}=17)$ \\
Ventricular extrasystole & $11.67 \%(\mathrm{n}=21)$ \\
Atrial fibrillation & $25 \%(\mathrm{n}=45)$ \\
Atrial flutter & $4.44 \%(\mathrm{n}=8)$ \\
Ventricular tachycardia & $5.56 \%(\mathrm{n}=10)$ \\
Ventricular fibrillation & $5.00 \%(\mathrm{n}=9)$ \\
Type of conduction abnormalities & \\
Atrioventricular block & $8.89 \%(\mathrm{n}=16)$ \\
Left bundle branch block & $2.78 \%(\mathrm{n}=5)$ \\
Right bundle branch block & $3.89 \%(\mathrm{n}=7)$ \\
\hline
\end{tabular}




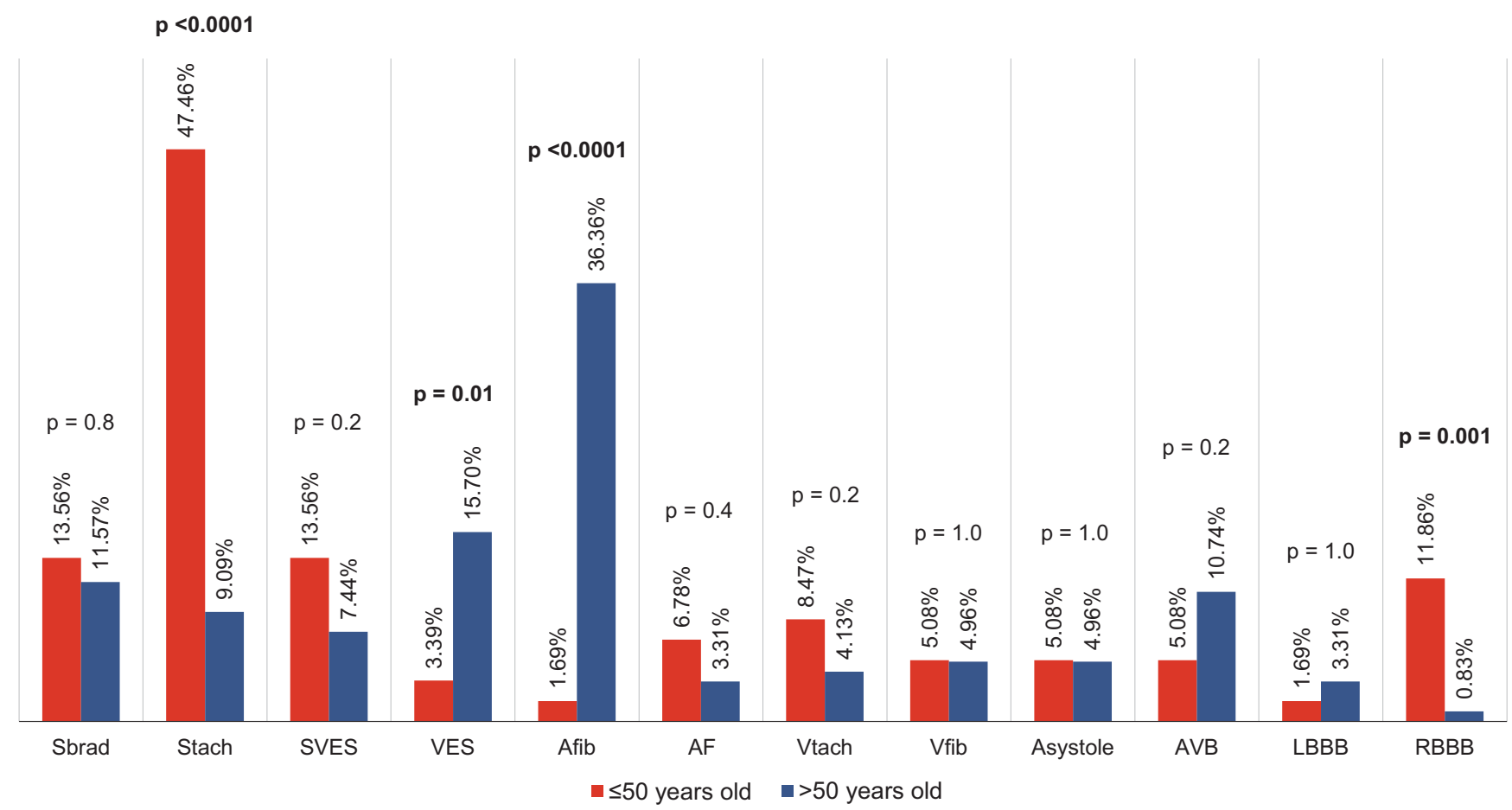

FIGURE 1. Rhythm and conduction abnormalities according to age groups. Sbrad - sinus bradycardia, Stach - sinus tachycardia, SVES supraventricular extrasystole, VES - ventricular extrasystole, Afib - atrial fibrillation, AF - atrial flutter, VTach - ventricular tachycardia, Vfib - ventricular fibrillation, AVB - atrioventricular block, RBBB - right bundle branch block, LBBB - left bundle branch block

nificantly higher percentage sinus tachycardia $(\mathrm{p}<0.0001)$ and right bundle branch block $(\mathrm{p}=0.001)$ (Figure 1$)$.
There were no statistically significant differences between patients from urban versus rural areas regarding

TABLE 2. Rhythm and conduction abnormalities in association with provenance and gender

\begin{tabular}{|c|c|c|c|c|c|c|c|c|c|c|}
\hline Analyzed parameter & $\begin{array}{c}\text { Rural } \\
(n=92)\end{array}$ & $\begin{array}{c}\text { Urban } \\
(n=88)\end{array}$ & $p$ value & $\begin{array}{l}\text { Odds } \\
\text { ratio }\end{array}$ & $95 \% \mathrm{CI}$ & $\begin{array}{c}\text { Female } \\
(n=99)\end{array}$ & $\begin{array}{c}\text { Male } \\
(\mathrm{n}=\mathbf{8 1})\end{array}$ & p value & $\begin{array}{l}\text { Odds } \\
\text { ratio }\end{array}$ & $95 \% \mathrm{CI}$ \\
\hline Bradycardia n (\%) & $13(14.1)$ & $9(10.2)$ & 0.4 & 1.44 & $0.53-4.06$ & $11(11.1)$ & $11(13.5)$ & 0.6 & 1.26 & $0.46-3.4$ \\
\hline Tachycardia n (\%) & $20(21.7)$ & $18(20.4)$ & 0.8 & 1.08 & $0.5-2.37$ & $21(21.2)$ & $17(20.9)$ & 0.9 & 1.01 & $0.46-2.23$ \\
\hline Atrial fibrillation $\mathrm{n}(\%)$ & $24(26.0)$ & $21(23.8)$ & 0.7 & 1.13 & $0.54-2.35$ & $26(26.26)$ & $19(23.4)$ & 0.6 & 1.16 & $0.56-2.45$ \\
\hline Atrial flutter n (\%) & $3(3.2)$ & $5(5.6)$ & 0.5 & 1.78 & $0.33-11.83$ & $4(4.0)$ & $4(4.9)$ & 1 & 1.23 & $0.22-6.84$ \\
\hline Ventricular tachycardia n (\%) & $3(3.2)$ & $7(7.9)$ & 0.2 & 2.55 & $0.56-15.8$ & $5(5.0)$ & $5(6.1)$ & 0.7 & 1.24 & $0.27-5.58$ \\
\hline Ventricular fibrillation $\mathrm{n}(\%)$ & $6(6.5)$ & $3(3.4)$ & 0.5 & 1.97 & $0.4-12.56$ & $5(5.0)$ & $4(4.9)$ & 1 & 1.02 & $0.21-5.35$ \\
\hline Right bundle branch block n (\%) & $4(4.3)$ & $3(3.4)$ & 1 & 1.29 & $0.21-9.04$ & $3(3.0)$ & $4(4.9)$ & 0.7 & 1.66 & $0.27-11.66$ \\
\hline Conduction abnormalities n (\%) & $16(17.3)$ & $12(13.6)$ & 0.5 & 1.33 & $0.55-3.31$ & $16(16.1)$ & $12(14.8)$ & 0.8 & 1.11 & $0.46-2.75$ \\
\hline Ischemic n (\%) & $7(7.6)$ & $8(9.0)$ & 0.7 & 1.21 & $0.37-4.13$ & $7(7.0)$ & $8(9.8)$ & 0.5 & 1.44 & $0.43-4.89$ \\
\hline Non-ischemic n (\%) & $23(25)$ & $15(17.0)$ & 0.2 & 1.62 & $0.74-3.63$ & $22(22.2)$ & $16(19.7)$ & 0.6 & 1.16 & $0.53-2.58$ \\
\hline
\end{tabular}




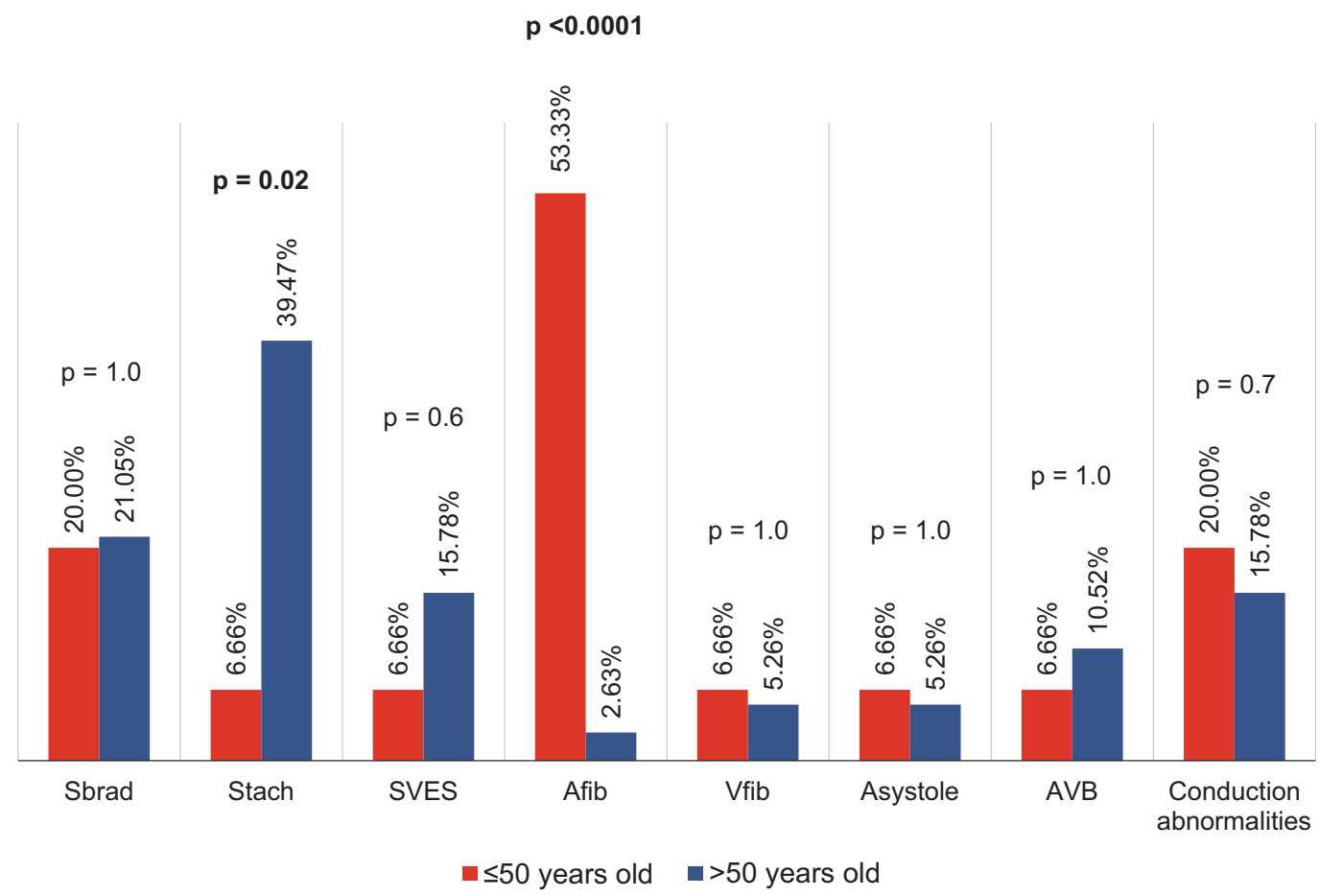

FIGURE 2. Comparison between ischemic and non-ischemic etiology of the rhythm and conduction abnormalities. Note: patients with unidentified cause of rhythm and conduction disturbances are not illustrated in the figure. Sbrad - sinus bradycardia, Stach - sinus tachycardia, SVES - supraventricular extrasystole, Afib atrial fibrillation, Vfib - ventricular fibrillation, AVB - atrioventricular block, Conduction abnormalities consist of any type of atrioventricular or interventricular conduction delay, diagnosed on the ECG

the type of arrhythmia or conduction disorders, or concerning the ischemic versus non-ischemic reason for requesting ambulance assistance (Table 2).

We found no statistically significant differences between males and females regarding the type of rhythm and conduction abnormalities, although we observed that females were more prone to present atrial fibrillation (Table 2).

\section{ETIOLOGY}

Regarding the etiology of the rhythm and conduction disturbances, in $70.56 \%(n=127)$ of cases there was no cause identified in prehospital settings. From the $29.44 \%$ of subjects with an identified etiology, 8.33\% $(n=15)$ had been diagnosed with acute myocardial infarction, while $21.11 \%(n=38)$ presented a non-ischemic etiology (endocrinological, neurological, trauma, electrolyte imbalance, intoxication, or anxiety).

There was a significantly higher number of $\mathrm{AF}$ in patients with acute myocardial ischemia $(\mathrm{p}<0.0001)$, whereas sinus tachycardia was more common among non-ischemic patients $(\mathrm{p}=0.02)$ (Figure 2$)$.

\section{SYSTEM-RELATED DIFFERENCES BETWEEN TERTIARY AND NON-TERTIARY BASE STATION HOSPITALS}

There were no statistically significant differences between the type of arrhythmia diagnosed in the ambulance, in emergency settings, in regions assisted by a tertiary hospital and the type of arrhythmia identified in regions affiliated to a non-tertiary medical center. This indicates a similar operating system of emergency care in both models of system of care (Table 3).

\section{DISCUSSIONS}

Prehospital ECG allows early diagnosis of life-threatening conditions, including ACS and cardiac arrhythmias. ${ }^{21,22} \mathrm{Ob}-$ taining a 12-lead ECG tracing in prehospital settings is of extreme importance for the diagnosis and effective management of such patients. A study conducted by Rawshani et al. on 13,151 patients showed that obtaining a pre-hospital ECG tracing leads to a significant decrease in 30-day mortality, especially in patients complaining of chest pain..$^{23}$

The results of our study revealed that from the total study population, $92.78 \%$ of patients complaining of the 
TABLE 3. Comparative analysis of arrhythmias and conduction abnormalities diagnosed in the mobile emergency unit between two regions served by a tertiary vs. non-tertiary hospital

\begin{tabular}{lccccc}
\hline Type of base station hospital & $\begin{array}{c}\text { Tertiary } \\
(\mathbf{n = 9 0 )}\end{array}$ & $\begin{array}{c}\text { Non-tertiary } \\
\mathbf{( n = 9 0 )}\end{array}$ & p value & Odds ratio & 95\% CI \\
\hline Bradycardia n (\%) & $9(10)$ & $13(14.44)$ & 0.3 & 1.52 & $0.56-4.27$ \\
Tachycardia n (\%) & $18(20)$ & $20(22.22)$ & 0.7 & 1.14 & $0.52-2.5$ \\
Supraventricular extrasystole n (\%) & $9(10.00)$ & $8(8.89)$ & 0.8 & 1.14 & $0.37-3.57$ \\
Ventricular extrasystole n (\%) & $11(12.22)$ & $10(11.11)$ & 0.8 & 1.11 & $0.4-3.11$ \\
Atrial fibrillation n (\%) & $22(24.44)$ & $23(25.56)$ & 0.8 & 1.06 & $0.51-2.21$ \\
Atrial flutter n (\%) & $3(3.33)$ & $5(5.56)$ & 0.7 & 1.7 & $0.32-11.3$ \\
Ventricular tachycardia n (\%) & $4(4.44)$ & $6(6.67)$ & 0.5 & 1.5 & $0.44-5.14$ \\
Ventricular fibrillation n (\%) & $5(5.56)$ & $4(4.44)$ & 1 & 1 & $0.26-6.59$ \\
Asystole n (\%) & $5(5.56)$ & $4(4.44)$ & 1 & 1.26 & $0.26-6.59$ \\
Atrioventricular block n (\%) & $9(10.00)$ & $7(7.78)$ & 0.6 & 1.32 & $0.41-4.37$ \\
Left bundle branch block n (\%) & $3(3.33)$ & $2(2.22)$ & 1 & 1.51 & $0.17-18.53$ \\
Right bundle branch block n (\%) & $4(4.44)$ & $3(3.33)$ & 1 & 1.35 & $0.22-9.46$ \\
Conduction abnormalities n (\%) & $16(17.78)$ & $12(13.33)$ & 0.4 & 1.4 & $0.58-3.49$ \\
Acute myocardial infarction n (\%) & $8(8.89)$ & $7(7.78)$ & 0.7 & 1.16 & $0.35-3.93$ \\
Non-cardiovascular pathologies n (\%) & $18(20.00)$ & $20(22.22)$ & 0.7 & 1.14 & $0.52-2.5$ \\
\hline
\end{tabular}

previously mentioned symptoms presented a type of arrhythmia, and $15.56 \%$ were diagnosed with an atrioventricular or interventricular conduction delay. The most frequent arrhythmia in the studied lot was AF (25\%), followed by sinus tachycardia, while the most frequent conduction delay was found to be AVB (8.89\%).

A study conducted by Brunetti et al. analyzed the prevalence of arrhythmias occurring in prehospital settings, on a population of 4 million inhabitants in South-Western Italy; tele-ECG tracings were recorded on 2,648 patients, who underwent remote interpretation by a specialized cardiologist. The study found that the most frequent type of arrhythmia was sinus tachycardia (13\%), while in case of bradycardia, the underlying ECG diagnosis was AVB, thus showing similarities to the results of our study. ${ }^{7}$

The prevalence of $\mathrm{AF}$ is higher with age, and it is the most frequently encountered arrhythmia over the age of 65, mainly due to the aging process of the heart, which leads to myocardial fibrosis and remodeling. 24,25 Ventricular extrasystole or premature ventricular beats increase in prevalence with increasing age, in patients with or without heart disease, fact that could also be explained by the structural and electrical remodeling process of the myocardium that occurs with age. ${ }^{3}$ When analyzing the study subjects by age groups, we found that older patients presented more frequent $\mathrm{AF}$ and ventricular extrasystole. The elderly present a significantly higher risk of arrhythmias due to the age-related physiological remodeling process of the myocardium, as well as to cardiovascular disease that is more frequent in this category of patients. The underlying ischemic heart disease and arterial hypertension lead to an increased mortality and morbidity of arrhythmias in geriatric patients. ${ }^{26,27} \mathrm{~A}$ study on the prevalence of palpitation, arrhythmias, and associated cardiovascular risk factors in ambulatory patients aged between 60-94 years, found that AF was the most common sustained arrhythmia in this age group population, followed by premature atrial and ventricular beats. In the same study, it was shown that patients with rhythm disturbances were more commonly males, significantly older, and with an increased rate of cardiovascular disease and chronic tobacco use compared to patients without arrhythmias. ${ }^{28}$

At the same time, in our study, patients younger than 50 years were more prone to be diagnosed with sinus tachycardia and right bundle branch block. In contradiction to our results, a study conducted on 855 men aged 50 years, who were followed up for the next 30 years, showed that the prevalence of RBBB was increasing with age ( $1 \%$ at the age of 50 years, to $17 \%$ at the age of 80 years), but no relation between RBBB and ischemia or mortality could be established. However, the study found that men with RBBB had an increased heart volume at 50 years, and had developed diabetes and congestive heart failure during the follow-up period, thus suggesting that RBBB is a marker for the gradual degenerative process of the myocardium. ${ }^{29}$ The Framingham study on RBBB, with a follow-up of 18 years, showed a significantly higher rate of coronary ar- 
tery disease and congestive heart failure, as well as an increased mortality in patients with RBBB compared to matching controls. ${ }^{30}$ Also, a study published in 2016 on the prognostic value of bundle branch blocks in survivors of an out of hospital cardiac arrest (OHCA), found that despite being transient in most cases, RBBB was directly associated with increased mortality and worse neurological outcome after OHCA. ${ }^{31}$

Our study found no statistically significant differences in the arrhythmias diagnosed in prehospital settings between the two genders. However, AF, sinus tachycardia, ventricular extrasystole, and AVBs were more frequently encountered in female patients, while male patients were more prone to present sinus bradycardia, ventricular tachycardia, and right bundle branch blocks. It is well known that there are important differences between males and females in the clinical characteristics of many cardiovascular diseases, which are also extended for rhythm and conduction disturbances, due to various hormonal and constitutional differences. ${ }^{32-34}$ The effects of sex hormones on the conductibility and excitability of the heart vary between genders, as testosterone leads to a shortening of the QT interval, while estrogen leads to an elongation of the QT interval. ${ }^{35} \mathrm{~A}$ study on 5,116 patients found that the resting heart rate of female patients is higher with up to 3-5 beats per minute. ${ }^{36}$ The gender distribution of arrhythmias has been previously described, where inappropriate sinus tachycardia, atrioventricular nodal reentry tachycardia, sinus node disease, and acquired or congenital long QT syndromes show a female predominance, while AV blocks, supraventricular extrasystole, AF, ventricular tachycardia, or sudden cardiac death are more frequent in men. ${ }^{37}$

The Reykjavik study on the epidemiology of right bundle branch blocks, based on a population of 9,135 men and 9,627 women, found an almost 2 -fold increase in the rate of RBBB diagnosed in men, with an age-dependent increase in the rate of diagnosis. ${ }^{38}$

Also, there were no statistically significant differences between the studied parameters when comparing the rural versus urban provenance, suggesting that the study population is homogenous, with similar geographical and demographical characteristics.

One of the secondary objectives of the study was to evaluate the etiology of rhythm and conduction disturbances, which could be diagnosed in prehospital settings. We observed that in $70.56 \%$ of patients a clear cause could not be identified. These subjects required further diagnostic investigations performed after transportation to the base station hospital. However, in $29.44 \%$ of patients, the etiology was found to be of either ischemic or non-ischemic origin. The non-ischemic etiology included patients with conditions that could cause secondary arrhythmias, but in whom myocardial ischemia was excluded by the ECG tracings, more specifically subjects with anxiety, trauma, intoxications, endocrinological or neurological disorders, or electrolyte imbalances.

ACSs have an increased risk for benign or malignant arrhythmias, both in the acute phase and on the long-term, due to reentry circuits developed around the ischemic scar tissue. SCD has decreased in the acute phase of ACS due to timely application of revascularization procedures, however many SCDs occur in the prehospital phase. ${ }^{12,15}$ Following an episode of acute ischemia, the myocardium undergoes a process of structural and functional remodeling, becoming a favorable terrain for ventricular tachycardias, which lead to increased mortality both in the early and late phase of the acute event. ${ }^{39}$

The most frequent supraventricular arrhythmia in STEMI patients is AF, having an incidence of $21 \%$ including preexistent, newly diagnosed, or first time onset $\mathrm{AF} .4^{0}$ The presence of AF in STEMI patients leads to a higher reinfarction rate and an increased risk of developing congestive heart failure, stroke, or SCD. ${ }^{41-43}$ Bradyarrhythmias associated with acute myocardial ischemia include sinus bradycardia occurring in the first hours of STEMI, especially in inferior localization of the infarction, and AVBs. ${ }^{15,44}$ In general, AVBs associated with infarction of the inferior wall resolve after revascularization. However, those associated with anterior infarctions, caused by an extensive necrosis of the anterior wall, are associated with higher mortality rates. ${ }^{15}$ In patients with acute myocardial infarction, development of a new bundle branch block, right or left, is associated with higher short- and long-term mortality rates. 45

Non-cardiac etiologies of rhythm and conduction abnormalities include electrolyte imbalances, psychiatric disorders and antipsychotic drugs, peripartum cardiomyopathy, neurological disorders such as epilepsy, neuromuscular disorders, as well as illegal recreation drug use that leads to electrical instability of the myocardium..$^{46-49}$

In our study, we compared the features of rhythm and conduction abnormalities between patients with acute myocardial ischemia and those with non-ischemic origin of the arrhythmia. The most frequently diagnosed ischemic arrhythmia was found to be AF, while patients without acute myocardial ischemia were diagnosed mainly with sinus tachycardia, followed by sinus bradycardia, supraventricular extrasystole, and AVB.

Another objective of our study was to perform a comparative analysis between patients located in a region served by a tertiary versus non-tertiary base station hospital. A 
tertiary hospital is a specialized medical center, usually as a referral location for primary and secondary care medical personnel, which detains advanced and complex diagnostic and therapeutic facilities, while a non-tertiary medical center comprises primary and secondary care..$^{50}$

We included patients from two neighboring counties in Central Romania (Cluj and Sălaj), which are served by a tertiary and non-tertiary base station hospital. The reason for choosing two neighboring counties was to perform a comparative analysis on the rhythm and conduction abnormalities diagnosed in the ambulance, between a region served by a tertiary versus a non-tertiary hospital, in a population with similar demographical and geographical characteristics. Cluj is a region assisted by an academic hospital, with several advanced possibilities for diagnosis and treatment, including a very well-developed system for emergency assistance, being also a PCI center that serves the northern and eastern part of Romania. Sălaj on the other hand, is served by a non-tertiary hospital, offering emergency medical assistance and other specialized medical services, having Cluj County Hospital as tertiary referral center in complex cases.

Our results showed a similar operating system of emergency care in both models of system of care, with no statistically significant differences between the regions served by a tertiary and non-tertiary medical center regarding the diagnosis of rhythm and conduction abnormalities in emergency care units. Despite all the advanced diagnostic facilities of a tertiary hospital, there were no significant differences regarding the prehospital approach of a patient; this illustrates a well-functioning emergency system regardless of the geographical location.

\section{CONCLUSIONS}

The present study showed that the most frequently diagnosed arrhythmia in prehospital settings, for patients requesting the ambulance for symptoms characteristic for a rhythm or conduction abnormality, was $\mathrm{AF}$ and sinus tachycardia, while AVBs were the most frequent conduction disturbances. Young patients more frequently present sinus tachycardia or right bundle branch blocks, while older subjects are more prone to develop ischemia-related $\mathrm{AF}$ and ventricular extrasystole. In most cases, a clear etiology of the arrhythmia could not be identified by only using the equipment provided by the mobile emergency medical unit, and these patients required further investigations in the base station hospital. There were no differences in rhythm and conduction abnormalities diagnosed in the ambulance, in patients from regions that are served by a tertiary or a non-tertiary medical center, indicating an efficient emergency system of care regardless of the geographical region.

\section{CONFLICT OF INTEREST}

Nothing to declare.

\section{REFERENCES}

1. Granfeldt A, Wissenberg M, Hansen SM, et al. Resuscitation. 2016;108:40-47. doi: 10.1016/j.resuscitation.2016.08.024.

2. Pätz T, Wolfrum S. Tachycardic Arrhythmias in the Emergency Room - State of the Art. Dtsch Med Wochenschr. 2017;142:1118 1122. doi: 10.1055/s-0043-110490.

3. Chow GV, Marine JE, Fleg JL. Epidemiology of Arrhythmias and Conduction Disorders in Older Adults. Clinics in geriatric medicine. 2012;28:539-553. doi: 10.1016/j.cger.2012.07.003.

4. Trappe HJ. Concept of the five "A"s for treating emergency arrhythmias. Journal of Emergencies, Trauma and Shock. 2010;3:129-136. doi: 10.4103/0974-2700.62111.

5. Bosson N, Kaji AH, Niemann JT, et al. The Utility of Prehospital ECG Transmission in a Large EMS System. Prehosp Emerg Care. 2015;19:496-503. doi: 10.3109/10903127.2015.1005260.

6. Morrison LJ, Brooks S, Sawadsky B, et al. Pre-hospital 12-lead electrocardiography impact on acute myocardial infarction treatment times and mortality: a systematic review. Acad Emerg Med. 2006;13:84-89. doi: 10.1197/j.aem.2005.07.042.

7. Brunetti ND, De Gennaro L, Dellegrottaglie G, Antonelli G, Amoruso D, Di Biase M. Prevalence of cardiac arrhythmias in pre-hospital tele-cardiology electrocardiograms of emergency medical service patients referred for syncope. J Electrocardiol. 2012;45:727-732. doi: 10.1016/j.jelectrocard.2012.07.018.

8. Zègre Hemsey JK, Drew BJ. Prehospital electrocardiography: a review of the literature. J Emerg Nurs. 2012;38:9-14. doi: 10.1016/j.jen.2011.09.001.

9. Bobinger T, Kallmünzer B2, Kopp M, et al. Diagnostic value of prehospital ECG in acute stroke patients. Neurology. 2017;88:1894-1898. doi:10.1212/WNL.0000000000003940.

10. Josephson M, Wellens HJ. Implantable defibrillators and sudden cardiac death. Circulation. 2004;109:2685-2691. https://doi.org/10.1161/01.CIR.0000129322.97266.F3.

11. Eckart RE, Shry EA, Burke AP, et al. Sudden death in young adults: an autopsy-based series of a population undergoing active surveillance. J Am Coll Cardiol. 2011;58:1254-1261. doi: 10.1016/j.jacc.2011.01.049.

12. Priori SG, Bloomstrom-Lundqvist C, Mazzanti A, et al. 2015 ESC Guidelines for the management of patients with ventricular arrhythmias and the prevention of sudden cardiac death. Eur Heart J. 2015; 36:2793-2867. doi: 10.1093/eurheartj/ehv316.

13. Maron BJ. Sudden death in young athletes. N Engl J Med. 2003;349:1064-1075. doi: 10.1056/NEJMra022783.

14. Van Camp SP, Bloor CM, Mueller FO, Cantu RC, Olson HG. Nontraumatic sports death in high school and college athletes. Med Sci Sports Exerc. 1995;27:641-647.

15. Ibanez B, James S, Agewall S, et al. 2017 ESC Guidelines for the management of acute myocardial infarction in patients presenting with ST-segment elevation. Eur Heart J. 2017;00:166. doi:10.1093/eurheartj/ehx393.

16. Ghuran A, Nolan J. Recreational drug misuse: issues for the cardiologist 16. Heart. 2000;83:627-633. 
17. Ismailov RM, Ness RB, Redmond CK, Talbott EO, Weiss HB. Trauma Associated with Cardiac Arrhytmias: Results From a Large Matched Case-Control Study. J Trauma. 2007;62:11861191. doi: 10.1097/01.ta.0000215414.35222.bb.

18. Seemann A, Boissier F, Razazi K, et al. New-onset supraventricular arrhythmia during septic shock: prevalence, risk factors and prognosis. Annals of Intensive Care. 2015;5:27. doi:10.1186/s13613-015-0069-5.

19. Schwartz A, Brotfain E, Koyfman L, et al. Association between Hypophosphatemia and Cardiac Arrhythmias in the Early Stage of Sepsis: Could Phosphorus Replacement Treatment Reduce the Incidence of Arrhythmias? Electrolytes \& Blood Pressure: E \& BP. 2014;12:19-25. doi: 10.5049/EBP.2014.12.1.19.

20. Cecchi E, Grossi F, Rossi M, Giglioli C, De Feo ML. Severe hypocalcemia and life-threatening ventricular arrhythmias: case report and proposal of a diagnostic and therapeutic algorithm. Clinical Cases in Mineral and Bone Metabolism. 2015;12:265-268. doi: 10.11138/ccmbm/2015.12.3.265.

21. Kudenchuk PJ, Redshaw JD, Stubbs BA, et al. Impact of changes in resuscitation practice on survival and neurological outcome after out-of-hospital cardiac arrest resulting from nonshockable arrhythmias. Circulation. 2012;125:1787-1794. doi: 10.1161/CIRCULATIONAHA.111.064873.

22. Boothroyd LJ, Segal E, Bogaty $P$, et al. Information on myocardial ischemia and arrhythmias added by prehospital electrocardiograms. Prehosp Emerg Care. 2013;17:187-92. doi: 10.3109/10903127.2012.755583.

23. Rawshani N, Rawshani A, Gelang C, et al. Association between use of pre-hospital ECG and 30-day mortality: A large cohort study of patients experiencing chest pain. Int J Cardiol. 2017;248:77-81. doi: 10.1016/j.ijcard.2017.06.032.

24. Karamichalakis N, Letsas KP, Vlachos K, et al. Managing atrial fibrillation in the very elderly patient: challenges and solutions. Vascular Health and Risk Management. 2015;11:555562. doi: 10.2147/VHRM.S83664.

25. Go AS, Hylek EM, Phillips KA, et al. Prevalence of diagnosed atrial fibrillation in adults: national implications for rhythm management and stroke prevention: the AnTicoagulation and Risk Factors in Atrial Fibrillation (ATRIA) Study. JAMA. 200; 285:2370-2375.

26. Lasses y Ojeda LA. Heart arrhythmia in the elderly. Arch Cardiol Mex. 2002;72 Suppl 1:S106-110.

27. Sakabe K1, Fukuda N, Nada T, et al. Age-related changes in the electrophysiologic properties of the atrium in patients with no history of atrial fibrillation. Jpn Heart J. 2003;44:385-393.

28. Lok NS, Lau CP. Prevalence of palpitations, cardiac arrhythmias and their associated risk factors in ambulant elderly. Int J Cardiol. 1996;54:231-236.

29. Eriksson P, Hansson PO, Eriksson H, Dellborg M. Bundlebranch block in a general male population: the study of men born 1913. Circulation. 1998;98:2494-500.

30. Schneider JF, Thomas HE, Kreger BE, McNamara PM, Sorlie P, Kannel WB. Newly acquired right bundle-branch block: The Framingham Study. Ann Intern Med. 1980;92:37-44.

31. Grand J, Thomsen JH, Kjaergaard J, et al. Prevalence and $\underline{\text { Prognostic Implications of Bundle Branch Block in Comatose }}$ Survivors of Out-of-Hospital Cardiac Arrest. Am J Cardiol. 2016;118:1194-1200. doi: 10.1016/j.amjcard.2016.07.034.

32. Gowd BM, Thompson PD. Effect of female sex on cardiac arrhythmias. Cardiol Rev. 2012;20:297-303. doi: 10.1097/ CRD.ob013e318259294b.
33. Larsen JA, Kadish AH. Effects of gender on cardiac arrhythmias. J Cardiovasc Electrophysiol. 1998;9:655-664.

34. Yarnoz MJ, Curtis AB. More reasons why men and women are not the same (gender differences in electrophysiology and arrhythmias). Am J Cardiol. 2008;101:1291-126. doi:10.1016/j. amjcard.2007.12.027.s.

35. Nakagawa $M$, Ooie $\mathrm{T}$, Takahashi $\mathrm{N}$, et al. Influence of menstrual cycle on QT interval dynamics. Pacing Clin Electrophysiol 2006;29:607-613. doi: 10.1111/j.1540-8159.2006.00407.x.

36. Liu K, Ballew C, Jacobs DR Jr, et al. Ethnic differences in blood pressure, pulse rate, and related characteristics in young adults. The CARDIA study. Hypertension 1989;14:218-226.

37. Linde C. Women and arrhythmias. Pacing Clin Electrophysiol 2000;23:1550-1560.

38. Thrainsdottir IS, Hardarson T, Thorgeirsson G, Sigvaldason $\mathrm{H}$, Sigfusson N. The epidemiology of right bundle branch block and its association with cardiovascular morbidity--the Reykjavik Study. Eur Heart J. 1993;14:1590-1596.

39. Ajijola OA, Tung R, Shivkumar K. Ventricular tachycardia in ischemic heart disease substrates. Indian Heart Journal. 2014;66:S24-S34. doi:10.1016/j.ihj.2013.12.039.

40. Schmitt J, Duray G, Gersh BJ, Hohnloser SH. Atrial fibrillation in acute myocardial infarction: a systematic review of the incidence, clinical features and prognostic implications. Eur Heart J. 2009;30:1038-1045. doi: 10.1093/eurheartj/ehn579.

41. Batra G, Svennblad B, Held C, et al. All types of atrial fibrillation in the setting of myocardial infarction are associated with impaired outcome. Heart. 2016;102:926-933. doi:10.1136/ heartjnl-2015-308678.

42. Jabre P, Jouven X, Adnet F, et al. Atrial fibrillation and death after myocardial infarction: a community study. Circulation. 2011;123:2094-2100. https://doi.org/10.1161/ CIRCULATIONAHA.110.990192.

43. Siu CW, Jim MH, Ho HH, et al. Transient atrial fibrillation complicating acute inferior myocardial infarction: implications

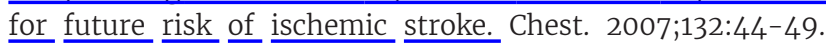
doi:10.1378/chest.06-2733.

44. Chen A, Ashburn MA. Cardiac effects of opioid therapy. Pain Med. 2015;16:S27-31. doi: 10.1111/pme.12915.

45. Melgarejo-Moreno A, Galcerá-Tomás J, Consuegra-Sánchez L, et al. Relation of New Permanent Right or Left Bundle Branch Block on Short- and Long-Term Mortality in Acute Myocardial Infarction Bundle Branch Block and Myocardial Infarction. Am J Cardiol. 2015;116:1003-1009. doi:10.1016/j. amjcard.2015.07.019.

46. Nishimura M, Nakayama K, Ishikawa Y. Cardiac arrhythmias caused by electrolyte imbalance. Nihon Rinsho. 1996;54:22072212.

47. Jones ME, Campbell G, Patel D, et al. Risk of mortality (including sudden cardiac death) and major cardiovascular events in atypical and typical antipsychotic users: a study with the general practice research database. Cardiovasc Psychiatry Neurol .2013;2013:647476. doi: 10.1155/2013/647476.

48. Appleby L, Thomas S, Ferrier N, Lewis G, Shaw J, Amos T.

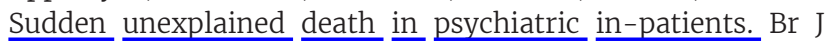
Psychiatry. 2000;176:405-406.

49. Ghuran A, Nolan J. The cardiac complications of recreational drug use. Western Journal of Medicine. 2000;173:412-415.

50. Tertiary Care Definition. Available at: https://www. hopkinsmedicine.org/patient_care/billing-insurance/ insurance_footnotes.html 